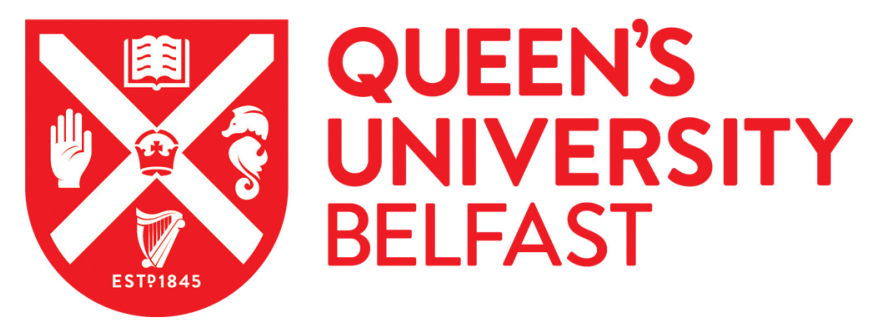

\title{
Does Trauma-Related Training Have a Relationship With, or Impact On, Mental Health Professionals' Frequency of Asking About, or Detection of, Trauma History? A Systematic Literature Review
}

Coyle, L., Hanna, D., Dyer, K. F. W., Read, J., Curran, D., \& Shannon, C. (2019). Does Trauma-Related Training Have a Relationship With, or Impact On, Mental Health Professionals' Frequency of Asking About, or Detection of, Trauma History? A Systematic Literature Review. Psychological Trauma: Theory, Research, Practice, and Policy. https://doi.org/10.1037/tra0000434

Published in:

Psychological Trauma: Theory, Research, Practice, and Policy

Document Version:

Peer reviewed version

Queen's University Belfast - Research Portal:

Link to publication record in Queen's University Belfast Research Portal

\section{Publisher rights}

(c) 2019 American Psychological Association. This work is made available online in accordance with the publisher's policies. Please refer to any applicable terms of use of the publisher.

\section{General rights}

Copyright for the publications made accessible via the Queen's University Belfast Research Portal is retained by the author(s) and / or other copyright owners and it is a condition of accessing these publications that users recognise and abide by the legal requirements associated with these rights.

Take down policy

The Research Portal is Queen's institutional repository that provides access to Queen's research output. Every effort has been made to ensure that content in the Research Portal does not infringe any person's rights, or applicable UK laws. If you discover content in the

Research Portal that you believe breaches copyright or violates any law, please contact openaccess@qub.ac.uk. 
Does Trauma-Related Training Have a Relationship with, or Impact on, Mental Health

Professionals Frequency of Asking About, or Detection of, Trauma History? A Systematic Literature Review

Lisa Coyle ${ }^{1}$, Donncha Hanna ${ }^{1}$, Kevin Dyer ${ }^{2}$, John Read ${ }^{3}$, David Curran ${ }^{1}$ and Ciaran Shannon ${ }^{2}$

${ }^{1}$ School of Psychology, Queen's University Belfast, Northern Ireland

${ }^{2}$ Department of Clinical Psychology, Northern Health and Social Care Trust Northern Ireland ${ }^{3}$ School of Psychology, University of East London, England

\section{Author Note}

This research was completed by the first author for the qualification of Doctorate in Clinical Psychology. There were no conflicts of interest or financial contributions that could have impacted the results of the study.

Correspondence should be addressed to Donncha Hanna, School of Psychology, DClinPsych, David Keir Building, 18-30 Malone Road, Belfast, BT9 5NP, Northern Ireland. Tel: 00442890 975549; Email: donncha.hanna@qub.ac.uk.

Word count: 7409 


\begin{abstract}
Objective: Exposure to traumatic events is prevalent, with the impact of trauma and its relationship with other conditions widely documented. Research suggests that clinicians do not routinely ask about trauma history in clinical settings. Trauma-related training has been recommended as a means of addressing this. Neither the impact of training on clinician behavior (i.e. frequency of asking about or detection of trauma history), nor the relationship between training and these variables, have been formally reviewed. Method: A systematic literature review was conducted using PsychINFO, Scopus and Web of Science. The grey literature and reference lists of included articles were consulted. Nine articles met the eligibility criteria for inclusion. Results: Two-thirds of studies reported statistically significant evidence to suggest that trauma related training is related to; (1) increased frequency of asking about trauma history and (2) greater detection of trauma history. Conclusions: Whilst acknowledging the limited number and variable quality of studies, as well as the failure to detect statistical significance in all studies, this review provides some evidence that trauma-related training is related to clinician inquiry and detection of trauma history. Further high quality research is needed. Training should address barriers to inquiry, acknowledge the potential variation in inquiry behaviour across trauma subtypes and encompass a skill and educational approach to both inquiry and response to trauma. Keywords: trauma history, trauma-related training, mental health professionals, asking about trauma, routine inquiry. Clinical impact statement: Mental health professionals do not routinely ask about trauma history and training has been recommended to address this. The findings of this review may have important implications with regards to the development and provision of future trauma-related training programmes for mental health professionals.
\end{abstract}


Does Trauma-Related Training Have a Relationship with, or Impact on, Mental Health Professionals Frequency of Asking About, or Detection of, Trauma History? A Systematic Literature Review

It is estimated that between $51 \%-84 \%$ of adults in the general population have been exposed to some type of traumatic event (Bernat, Ronfeldt, Calhoun \& Arias, 1998; Frans, Rimmo, Aberg \& Fredrikson, 2005; Kessler, Sonnega, Bromet, Hughes \& Nelson, 1995; Vrana \& Lauterbach, 1994). However, exposure to traumatic events in clinical populations tends to be higher (Elhai, Ford \& Naifeh, 2010), ranging between 76\%-91\% (Cusack, Freuh \& Brady, 2004; Wota et al., 2014). Traumatic exposure is associated with a range of mental health difficulties (Elhai., et al., 2010), most notably, PTSD (Bunting, Murphy, O’Neill \& Ferry, 2013).

While the importance of conducting a comprehensive assessment of trauma has been emphasized (Krinsley, Gallagher, Weathers, Kutter \& Kaloupek, 2003), there is a substantial body of evidence to suggest that mental health professionals do not frequently inquire about a history of traumatic exposure (Agar, Read \& Bush, 2002; Cunningham et al., 2016; Hepworth \& McGowan, 2013; Mitchell, Gatson-Grindel \& Laurenzano, 1996; Read, Harper, Tucker and Kennedy, 2018a; Rossiter et al., 2015; Shannon, Maguire, Anderson, Meenagh \& Mulholland, 2011). The low rates of inquiry about trauma history by mental health professionals is cause for concern, given that service users are more likely to disclose a trauma history when asked explicitly (Agar, Read \& Bush, 2002; Briere \& Zaidi, 1989; Read \& Fraser, 1998). Consequently, the failure to explicitly ask service users about their trauma history may lead to undetected trauma histories and have ramifications for assessment, formulation and intervention (Mitchell et al., 1996). 
Research has explored reasons for non-inquiry by professionals, including; concerns about offending or distressing the client, the need to prioritise more immediate concerns, concerns regarding vicarious trauma, fear of inducing false memories and a lack of training in asking about or responding to disclosures (Read, Hammersley \& Rudegeair, 2007). Others include confidence in asking about and responding to disclosures (Mansfield, Meehan, Forward \& Richardson-Clarke, 2017), clinician and client gender, client age and clinical diagnosis (Cunningham et al., 2016; Read et al., 2007; Shannon et al., 2011). The low rates of inquiry regarding trauma history by mental health professionals has led to a number of recommendations, including the development of guidelines, policies and training programmes to improve inquiry and response to trauma history disclosures (Cunningham et al., 2016; Mansfield et al., 2017; Read et al., 2018a; Rossiter et al., 2015; Shannon et al., 2011).

\section{Aim of current review}

Low rates of trauma history inquiry by mental health professionals have been met with a resounding recommendation that mental health professionals receive training to address this. In order to evaluate the utility of this training, it is important to ascertain if trauma-related training is related to, or has an impact on, mental health clinicians inquiry, or detection of, trauma history.

The primary aim of this systematic review is to consider if trauma-related training, delivered to mental health professionals, has a relationship with, or impact on, mental health clinician behavior (i.e. frequency of asking about or the detection of trauma history). Secondly, important components of training programmes will be identified in order to make recommendations for the designing of effective training programmes. 


\section{Method}

\section{Search strategy}

The protocol for this review was registered and published with prospero (registration number: CRD 42017077523). A literature search was conducted in November 2017, using Scopus, Web of Science and PsychINFO. The search terminology for Web of Science was as follows: training or program* and trauma or abuse or neglect or maltreatment or violence or "sexual assault" or "physical assault" or "domestic violence" or "intimate partner violence" or "sexual violence" and "mental health profession*" or "mental health staff" or "healthcare profession*" or psychiatric or "mental health service" and ask* or detect* or "training experience\$" and analys?s or survey or questionnaire or file or form or audit or evaluation or record* or simulation or "case vignette" or assess*. Subject headings were used in PsychINFO and combined using "or" with the key words above. A hand search of the grey literature was also conducted in November 2017 using Google scholar and also, via an online source ("Grey Literature Report", n.d.). No additional records were obtained.

A total of 1,283 publications were identified using database searches (424 from Scopus, 223 from Web of Science and 636 from PsycINFO. One thousand and ninety-six records remained following the removal of duplications. Abstracts and titles were screened according to eligibility criteria as follows:

i. Studies that evaluated the impact of trauma-related training on mental health clinicians inquiry behaviour. Studies investigating the relationship between trauma-related training and frequency of asking about trauma history or detection of trauma history.

ii. Participants who are mental health professionals, or professionals working in mental health services who work as part of a team/service to provide a mental health service. Studies 
including professionals who did not provide or work as part of a team or service delivering a mental health service were excluded.

iii. "Trauma-related training" was defined as training in the area of trauma which could be specifically related to specific types of trauma histories e.g. domestic violence, sexual abuse, or more general. Training could be part of the mental health professionals initial professional training, additional to professional training, or provided as part of a service initiative or training policy.

iv. The outcome measures for this review (i.e. frequency of asking about trauma history or detection of trauma history) could be captured by any means (e.g. professional self-report via survey, questionnaire, recorded in clinical files/notes or via service audits).

v. Published and unpublished studies in the form of journal articles, service related/research evaluations or reports and dissertations. Books, book chapters or other reviews were excluded. Studies published in English. Studies in other languages were excluded.

vi. Quantitative studies, including surveys or questionnaires incorporating open-ended questions and responses. Qualitative studies were excluded. Two qualitative studies were identified in the review process with neither of these studies addressing the question of this review.

Following completion of the screening process, 39 potentially suitable articles were identified and the full texts were then retrieved for full review. Thirty-two articles did not meet the eligibility criteria, leaving seven articles identified as meeting all criteria. The reference lists of articles meeting the inclusion criteria were then hand searched for additional articles. A further five full text papers were assessed for eligibility with two of these articles meeting the criteria for inclusion. Three international dissertations were also identified via the references of included 
texts. The authors of these dissertations were contacted and replies from two of the three corresponding authors confirmed that these dissertations would not have met the eligibility criteria; the third dissertation was not retrievable. The nine articles meeting the criteria were included in the review (Figure 1 Supplemental material). A second rater assessed eligibility for inclusion and Cohen's Kappa inter-rater reliability was calculated at .87, indicating strong agreement (McHugh, 2012). Any discrepancies were resolved through discussion.

Given the small number of articles meeting the inclusion criteria of the review, quality appraisal was conducted on the included articles in order to assess the quality of these studies, rather than to eliminate studies from the review. The quality appraisal tool incorporated items from the Quality Assessment Tool for Before-After (Pre-Post) Studies with No Control Group and the Quality Assessment Tool for Observational Cohort and Cross-Sectional Studies, as developed by the National Heart, Lung \& Blood Institute in 2014 (NHLBI, 2014a; NHLBI, 2014b). Items were also informed by STROBE guidelines (Strengthening the Reporting of Observational studies in Epidemiology; von Elm et al., 2007) and The Centre for Reviews and Dissemination (CRD, 2008) guidelines. See Supplementary material for quality tool.

Studies were assessed using nine items based on a three quality rating system informed by the Scottish Intercollegiate Guidelines Network guidance (SIGN, 2011). Quality was also assessed by a second rater and discrepancies were resolved through discussion. Total scores were used to inform an overall quality category for each individual study, using the NHLBI (2014) coding criteria of "good", "fair" or "poor". "Good" studies have the least amount of bias and results are considered valid, "fair" studies are susceptible to some bias, while "poor" studies have a significant risk of bias (NHLBI, 2014a; NHLBI, 2014b). Studies varied in their level of quality, with only one study identified as "good" (See Table 1 Supplementary material for scoring). 


\section{Results}

\section{Synthesis of results}

Table 2 (Supplementary material) provides a summary and key results of each study. Six of the nine studies included in this review set out with the overall aim to evaluate the effectiveness of a specific training intervention on various outcomes (Cavanagh et al., 2004; Currier \& Briere, 2000; Donohoe, 2010; Lotzin et al., 2018; Walters et al., 2016). One study aimed to ascertain if there had been any change in rates of recorded and identified abuse/neglect since the establishment of a training program and policy within a service (Sampson \& Read, 2017). Three studies examined training more generally with two of these studies examining training needs and experiences of mental health professionals (Currier et al., 1996; Murray et al., 2016) and the final study predominantly focused on identifying barriers in obtaining abuse histories (Young et al., 2001). With regards to design, three studies employed a cross-sectional design, five studies adopted an experimental/quasi-experimental approach, and one study was a survey evaluation.

With regards to participant demographics, participants, who were mental health professionals, $(N=780)$, ranged between $18-65$ years in age. The majority of professionals were female. Details on age and gender were not provided in three studies (Currier et al., 1996; Currier \& Briere, 2000; Walters et al., 2016) and the number of professionals in the Sampson \& Read (2017) study could not be determined due to the methodology (e.g. file analysis).

The nature and source of trauma-related training provided to clinicians varied across studies. In the six studies which focused on specific training interventions provided, training was in addition to professionals initial training (Cavanagh et al., 2004; Currier \& Briere, 2000; Donohoe, 2010; Lotzin et al., 2018; Walters et al., 2016, Sampson \& Read, 2017). In the 
remaining three studies where training experiences were examined more generally, little information was available with regards to the details of the training received (e.g. Currier et al., 1996; Murray et al., 2016; Young et al., 2001) due to the design of the studies.

Across all studies, training tended to be either trauma-generic or focused on specific traumatic experiences, such as domestic violence, sexual abuse or abuse histories. Some training programmes covered a culmination of traumatic experiences. The components of trauma-related training, where provided, varied across studies, but usually consisted of some educational content (e.g. summary of research findings regarding trauma/abuse, information and guidance on various aspects of trauma including assessment/inquiry). Some studies also incorporated a focus on practical skill development in asking about trauma history and responding to disclosures (Cavanagh et al., 2004; Donohoe, 2010; Lotzin et al., 2018; Sampson \& Read, 2017) and one study also incorporated information regarding trauma interventions (Walters et al., 2016). Additionally, training tended to be delivered in group format, with the duration of training ranging from a one hour orientation to a full day programme with refresher session.

In total, four of the nine studies used surveys or self-report questionnaires to examine clinician frequency of asking about or detection of trauma history (Lotzin et al., 2018; Cavanagh et al., 2004; Donohoe, 2010; Walters et al., 2016). One study (Young et al., 2001) employed a case vignette. Two studies (Currier et al, 1996; Murray et al., 2016) used self-report questionnaires to measure both clinician inquiry behavior and capture training experiences simultaneously. Of the remaining two studies, one study compared trauma history detection rates obtained using a standardised trauma interview (Currier \& Briere, 2000) while the final study employed a file audit (Sampson \& Read, 2017). A diverse range of analytic methodologies were employed across the studies, including; t-tests, descriptive statistics, chi-square analyses, linear 
mixed modelling, as well as a correlational design. The first author of this review calculated the effect sizes for studies where this had not been conducted. A narrative synthesis of the key results of the studies relevant to the research question is explored using the following headings: specified trauma-related training programmes and generalized trauma-related training experiences.

\section{Specified trauma-related training programmes.}

\section{New Zealand training programme/"Learning how to ask training"}

Three of the nine studies included in this review either focused on or provided an evaluation of the New Zealand training programme. This was provided in addition to professional clinical training. The programme initially focused predominantly on physical and child sexual abuse, with the aim to improve clinician inquiry and response to trauma and abuse histories (Cavanagh et al., 2004). This one-day group training programme encompassed educational components, including; research summaries of the relationship between abuse and mental health, the prevalence and effects of abuse, research demonstrating how often mental health professionals are asking about abuse and facilitated discussions reflecting on the advantages and disadvantages of asking about abuse histories. The training also included practical skills in inquiry and responding to disclosures (Cavanagh et al., 2004).

Cavanagh et al. (2004) employed a self-report questionnaire, which was administered immediately prior to the training and six weeks post training $(N=85)$. A statistically significant increase in confidence in asking about abuse and in responding to disclosures was observed. Sixty-seven percent of participants reported that training had changed their clinical practice. A small number of participants provided some examples of specific changes in this regard $(n=17)$, which included "now I ensure that either myself or someone else working with the client asks 
about abuse" and "I tend to ask the question quite early on in therapy" (Cavanagh et al., 2004, pp. 141). Participants reported that in $64 \%$ of client cases, they believed they knew whether or not clients had an abuse history. There was no statistically significant change in the number of client cases in which clinicians believed that they knew whether or not there was an abuse history, suggesting that the training did not have a statistically significant impact on self-reported detection. It was not possible to calculate an effect size for this particular finding as the statistical information could not be retrieved.

The failure to detect statistically significant results may have been contributed to by a number of factors, including the small number of participants for whom pre and post-data were available $(n=31)$, as well as an insufficient time period in which to expect a change in detection (six weeks). Alternatively, it may be that participants were already demonstrating a high level of skill in inquiry prior to the delivery of the training programme, given that $33 \%$ of participants in the study had received additional training prior to the intervention in this regard. Cavanagh et al. (2004) acknowledged these limitations. Findings in this study also indicated that client diagnosis, gender and age of client continued to influence a small number of professionals decision as to whether or not to ask clients about their abuse history post-training.

Sampson and Read (2017) compared detection rates of sexual and physical abuse to those obtained in a previous audit (see Agar et al., 2002), which had been undertaken in 1997 prior to the introduction of a policy and requirement that mental staff attend training on inquiring about childhood trauma (i.e. the New Zealand training programme). At the time of this study, the training programme had been running a number of times per year for approximately nine years.

Following an audit of 250 randomly selected files from four community health centres, the authors found a statistically significant increase in rates of recorded child sexual and physical 
abuse, and adult sexual abuse, but no statistically significant increases in recorded rates of adult physical assault were observed. The author of this review calculated effect sizes using phi coefficients, revealing small effect sizes with regards to detection of child sexual and physical abuse and adult sexual assault, and a very small effect size in relation to adult physical assault. Additionally, female clinicians were statistically more likely to ask about trauma history at initial assessment than males and clients with psychosis were less likely to be asked. Despite the significant improvements, $34.8 \%$ of the audited client files revealed no indication of having been asked about trauma history by a professional at any stage of treatment. Findings need to be considered in relation to the difficulty attributing increased rates of recorded abuse (i.e. increased detection) directly to the training, given the potential for historical effects over the extended time period (approximately 20 years) and the inability to control for confounding variables.

Lotzin et al. (2018), using a cluster randomized control trial, investigated the impact of an adapted form of the New Zealand training programme referred to as the "Learning how to ask training" (Read et al., 2007) on a range of outcomes. The objective of the training was to increase clinicians frequency of asking about a range of traumatic experiences including neglect, physical, sexual and emotional abuse. In addition to the existing training components associated with the New Zealand training programme outlined previously, this one-day group training programme incorporated a refresher session three-months post-training. This consisted of discussions regarding clinicians' experiences of asking about and responding to trauma history disclosures, as well as a refresher on how to ask about trauma. Service providers were randomly allocated to either the intervention $(n=72)$ or control group $(n=76)$.

Using a self-reported questionnaire, participants who received the training reported a significantly greater change in frequency of asking about trauma history from baseline to three 
and six-month follow-up than those who were in the control group. A medium effect size estimate was observed in this regard. No significant change was observed between three and six month follow up suggesting that change was maintained (very small effect size estimate).

Statistically greater increases in knowledge about trauma, attitudes and confidence with regards to inquiry and response were observed in the intervention group in comparison to the control. The authors also found that whilst inquiry for all trauma types significantly increased for those who received the training programme, sexual abuse was asked about significantly less than other trauma types in both the intervention and control group. Gender, duration working in the centre, profession and previous trauma training, did not significantly predict change in frequency of asking about trauma. However, change was predicted by clinician age, with older clinicians more likely to have reported a change in frequency of asking. While the authors acknowledge various limitations associated with the study, such as the reliance on self-report questionnaires, this study achieved one of the highest quality ratings in this review.

\section{Other specific training programmes}

Donohoe (2010) provided an evaluation of the Victims of Violence and Abuse Prevention Programme in one of the pilot trusts $(N=53)$. This programme, which was provided in addition to professional training, focused on sexual abuse and aimed to equip clinicians with knowledge and skills in inquiry and response to disclosure. The training was delivered over one day in a group format and included educational components on sexual abuse as well as skill development for inquiry and response to sexual abuse history.

Using a self-report questionnaire administered post-training $(n=30)$ (no time period specified), $77 \%$ of mental health professionals agreed that the training had changed their clinical practice. Over $35 \%$ of participants reported that they were now asking about abuse history as part 
of their routine assessment. Participants also reported increased confidence, knowledge and awareness of abuse. Client diagnosis, age of client and gender was reported by some participants to influence their tendency to ask clients. However, the findings of this study are compromised by the small number of professionals who completed the questionnaire $(n=30)$, the reliance on descriptive statistics and the limited information reported regarding the questionnaire. Additionally, there may be some difficulty in attributing results to the training delivered, given that $50 \%$ of professionals in the study reported to have received prior training.

Sample size was also a concern in the Walters et al. (2016) study, which aimed to evaluate the effectiveness of a bespoke training programme developed in response to staffs' needs identified via a focus group $(N=14)$. This programme, which was additional to professionals initial training, aimed to improve knowledge about trauma (non-specified type), confidence in asking about and responding to trauma, in addition to reducing staff worries regarding assessment and treatment of trauma. The training consisted of a half-day programme, delivered in group format, and provided education on trauma (e.g. relationship with attachment, assessment and intervention strategies) as well as case discussion and problem solving.

Using a self-report questionnaire, no statistically significant change in the number of clients known to professionals with a trauma history was observed from pre-training (one month prior to training) to six-month follow-up. Similarly, there was no statistically significant selfreported change in professionals frequency of asking about trauma history from pre-training to follow up. However, the mean number of clients known to have a trauma history and the mean rate of inquiry about trauma history increased from pre to follow-up, and medium effect sizes were observed, as calculated by the author of this review. Given the effect sizes observed, failure to find statistically significant differences may be due to the limited data available for all time 
points $(n=7)$, a limitation acknowledged by the authors. However, it must be acknowledged that despite the limited sample, statistically significant improvements in staff confidence and knowledge in assessing and treating trauma were observed, in addition to increases in the number of clients receiving intervention for trauma. The authors also acknowledge the potential impact of staff restructuring on findings which occurred following the training.

Currier and Briere (2000) evaluated the impact of a brief trauma-related training intervention in a psychiatric emergency department. The intervention consisted of a one-hour trauma educational lecture on the prevalence of trauma, victimisation as well as the impact and assessment of trauma. Participants were randomly allocated to the intervention $(n=10)$ or to a control group $(n=8)$, where participants received no information on trauma. Using a standardised trauma interview, clinicians who received the trauma orientation detected significantly more trauma history (sexual, physical and total violence) than those in the control group. Follow-up analyses revealed that clinicians who received the orientation detected significantly more childhood sexual and physical abuse, but not childhood/adolescent peer sexual assault, adult spouse abuse or adult non-intimate physical assault, than those in the control group. Effect size calculations were calculated by the authors, which revealed small effect sizes for each of the above findings. In addition, female clinicians detected significantly more sexual violence than male clinician. However, neither clinician age nor education level had a significant effect on number of instances of sexual, physical or total violence detected per clinician. While findings led the authors to support the effectiveness of a brief trauma orientation on detection of trauma history, limitations included the small sample size $(N=18)$, insufficient details regarding time frame of the study and the lack of follow-up to ascertain if effects were maintained postintervention. 


\section{Generalized trauma-related training experiences}

The remaining studies in this review inquired about trauma-related training previously received by participants (i.e. exposure), predominantly via self-report questionnaire, and sought to establish whether this was associated with or related to clinician frequency of asking about or detection of trauma history.

Young et al. (2001) employed a self-report questionnaire and case vignette to investigate barriers to asking about abuse histories in a sample of Psychiatrists and Psychologists $(N=144)$. A survey item asked professionals if they had received training regarding abuse inquiry, to which $24 \%$ of professionals responded that they had not received training in this regard. The source of this training was not specified. Young et al. (2001) found that participants who had received training in abuse inquiry were significantly more likely to ask about abuse in the case vignette. The authors of this review calculated the effect size using Hedges g, which revealed a medium effect size in this regard. Neither clinician gender, nor years of clinical experience, profession or etiology beliefs, had a significant impact on probability of inquiry about abuse in the vignette. Results must be interpreted in light of the limited information obtained with regards to the content and format of training received by participants, as well as the simultaneous measurement of training exposure and clinician inquiry behavior.

Murray et al. (2016) distributed a self-report survey to mental health professionals to ascertain domestic violence training and experiences $(N=173)$. Over seventy-eight percent of professionals reported to have received domestic violence training and $94.8 \%$ of professionals reported to have received more general trauma-related training. The source of this training was not specified. Professionals who had received domestic violence training were statistically more likely to report screening all clients for domestic violence and screening for partner sexual abuse 
and trauma symptoms. Professionals with training also engaged in a statistically higher number of intervention practices, including inquiry about historical domestic violence. There were no significant differences between trained and untrained professionals with regards to the screening of clients for other types of trauma, or with regards to the tendency to screen for domestic violence or traumatic violence only if suspected. Effect size calculations, using phi coefficients, were conducted by the authors of this review and ranged from very small to small effect sizes for all findings.

Limitations of the study include the fact that the term "screening" was not defined by the questionnaire despite participants screening practices being under investigation which may have led variation in how participants interpreted and responded to this. The study also did not capture any information on training content and format. A final limitation is the simultaneous measurement of training exposure and clinician inquiry behavior.

With a similar objective, Currier et al. (1996) employed a self-report survey to identify the domestic training experiences and needs of psychiatric residents $(N=145)$. Twenty-eight percent reported to have received domestic violence training, either in medical school, residency or another source. The findings revealed that increased domestic violence detection in cases was significantly correlated with training in domestic violence and a medium effect size was observed. Additionally, $88 \%$ of those who reported to have had received training were significantly more likely to have detected six or more cases of domestic violence within the past year in comparison to just $48 \%$ of those who had not received training. A medium effect size between training and case detection was calculated by the authors of this review using phicoefficients. With regards to the cases of domestic violence detected, clinicians directly asked patients about domestic violence in $43 \%$ of cases. No correlation between case detection and 
clinician gender or length of post-graduate training was observed. Findings from this study should be interpreted with caution given the lack of information regarding training content and format, a lack of information regarding the survey development and design as well as simultaneous measurement of training exposure and clinician inquiry behavior.

\section{Discussion}

\section{Review of findings}

Two-thirds (6/9) of studies found statistically significant evidence to suggest that trauma training is related to; (1) increased frequency of asking about trauma history (Lotzin et al., 2018; Murray et al., 2016; Young et al., 2001) and (2) greater detection of trauma history (Currier \& Briere, 2000; Currier et al., 1996; Sampson \& Read, 2017). In addition, 35\% of mental health professionals in the Donohoe (2010) study reported that they were now asking about abuse history as part of their routine assessment. Two studies found no significant change in frequency of asking about trauma (Walters et al., 2016) or detection of trauma history, following training (Cavanagh et al., 2004). Effect sizes ranged from very small to medium. Overall, this review provides some evidence that trauma-related training is related to, or has an impact on, frequency of asking about, or detection of, trauma history. However, these findings need to be considered in light of the small number of studies, of varying and limited quality, included in this review.

Sample size was one of the main areas of concern for most, but not all of the six studies which directly evaluated or investigated specific training programmes. Indeed, the failure to detect statistically significant findings in the Cavanagh et al. (2004) and Walters et al. (2016) studies may have been contributed to by small sample size. Issues relating to sample size not only limit the statistical power of studies, but also limit the generalizability of findings and present a threat to the 
ecological validity of studies. For the three studies which examined trauma-related training experiences more generally, some of the main limitations of these studies were intrinsic to the study design (i.e. limited ability to control for the influence of other variables) and due to the focus of the study (i.e. lacking information regarding professionals training exposure).

With regards to data collection methods, most studies used self-report questionnaires to assess clinician inquiry, with three studies using questionnaire to measure both inquiry behavior and training simultaneously. Self-report may be distorted by memory and error, but also subject to social desirability bias (Donohoe, 2010; Lotzin et al., 2018; Walters et al., 2016). Furthermore, given that self-report measures were used to evaluate the impact of specific training programmes, this may have increased the potential risk for expectancy effects (Barker et al., 2010) and context effects (Breakwell, Hammond, Fife-Schaw \& Smith, 2006). Alternatives to self-report measures of inquiry or detection have been suggested in the literature, including service-related outcomes (i.e. monitoring service level case detection of trauma, the number of trauma-related interventions or referrals made for trauma interventions), as well as client based outcomes (Lotzin et al., 2018; Toner, Daiches \& Larkin, 2013).

\section{Recommendations for the designing of future training programmes}

The capacity to make recommendations to services or educators regarding future traumarelated programmes is challenging. This is largely due to the (1) small number of studies of varying quality, some of which did not report statistically significant findings, (2) the variation in training experiences across studies, and (3) the fact that not all studies in this review collected details regarding professionals' training experiences. Therefore, recommendations are tentative.

Professionals typically identified role plays as one of the most useful aspect of their training experience (Cavanagh et al., 2004; Donohoe, 2010; Lotzin et al., 2018). Other useful 
aspects of training include a clear structure to the training, an informal style of facilitation (Cavanagh et al., 2004), and the provision of written handouts and summaries (Cavanagh et al., 2004). Psychologists and Psychiatrists in the Young et al. (2001) study recommended that training also address inquiry techniques for different groups and the effects of child abuse and false allegations of abuse. Finally, more than one day is preferential with additional follow-up sessions (Cavanagh et al., 2004; Donohoe, 2010).

Findings in some studies of this review suggested that despite the provision of training, some trauma types were more likely to be asked about or detected than others. For example, Lotzin et al. (2018) observed that the frequency of asking about sexual abuse changed significantly less in both control and intervention groups in comparison to other trauma types. It may be that some trauma types are more difficult for professionals to ask about or detect than others and therefore, some may require more specific or additional attention in training.

Training should address the barriers to inquiry as highlighted in this review, including; gender, age, diagnosis, fear of inducing false memories and other attitudes beliefs. These echo the findings in the literature (Cunningham et al., 2016; Read et al., 2007; Shannon et al., 2011). With these barriers in mind, perhaps training could highlight the potential benefits of using trauma history measures to facilitate identification of traumatic exposure, which may offer a less intrusive and efficient means of assessing traumatic exposure for both client and clinician (Shannon et al., 2011; Rossiter et al., 2015). See Elhai, Gray, Kashdan and Franklin (2005) for a useful review on the most frequently used trauma exposure and symptoms measures.

It must be acknowledged that the detection of trauma history may not be sufficient to ensure appropriate treatment. In a recent systematic review by Read, Harper, Tucker and Kennedy (2018b), child abuse and neglect were incorporated into treatment plans in $12 \%-44 \%$ of 
cases and were included in formulations in $12 \%-57 \%$ of cases. Referrals for trauma therapeutic work were made in $8 \%-22 \%$ of cases. Responding to disclosures was included in some of the training programmes included in this review, and this is identified as an important component for future training programmes (Read et al., 2018b).

\section{Future directions for research and implications for clinical practice}

Further research is needed to determine the utility of delivering trauma-related training to mental health professionals with regards to the inquiry and detection of trauma history. Additionally, further research is necessary to determine what training programmes should comprise. High quality research designs, such as Lotzin et al. (2018), are needed in order to evaluate trauma-related training in a more robust and rigorous manner. Rujne et al. (2017) have published a protocol of a cluster randomised control trial designed to investigate the impact of domestic violence training on mental health professionals' detection of domestic violence. More objective outcomes of clinician inquiry behavior are recommended.

Whilst acknowledging the limitations of the included studies, this review provides some support regarding the utility of trauma-related training in relation to clinician inquiry behavior. Mental health professionals may benefit from such training, however, this needs to be encouraged and monitored. Following the implementation of a pilot programme in the UK, the Department of Health requested that professionals routinely ask about violence and abuse histories, in addition to attending the designated training to facilitate this (Brooker, Tocque, Kennedy \& Brown, 2016). In an evaluation of this policy, Brooker et al. (2016) found that only $66 \%$ of staff had received the recommended training, with only services users being asked about sexual/violent abuse in $17 \%$ in $2014 / 2015$. This suggests that without monitoring and perseverance from managing systems, the impact of training will be limited (Read et al., 2018a). 


\section{Strengths and weaknesses of the review}

This paper provides the first formal and systematic review of the literature examining trauma related training and clinician inquiry behaviour. The strength of this review is its contribution to the literature and the ability to put forward some recommendations, albeit tentatively, for the development of future training programmes. As mentioned previously, the small number of studies included, of varying and limited quality, is the primary weakness of this review.

\section{Conclusion}

This review provides some evidence that trauma-related training is related to; (1) increased frequency of asking about trauma history and (2) greater detection of trauma history. High quality research is needed. Findings suggest that training should address barriers to inquiry, acknowledge the potential variation in inquiry behaviour across trauma subtypes and encompass a skill and educational approach to both inquiry and response to trauma. 


\section{References}

Agar, K., Read, J., \& Bush, J. M. (2002). Identification of abuse histories in a community mental health centre: The need for policies and training. Journal of Mental Health, 11(5), 533543. doi:10.1080/09638230020023886

Barker, C., Pistrang, N. \& Elliott, R. (2010). Research methods in Clinical Psychology: An introduction for students and practitioners. Chichester; Wiley \& Sons.

Bernat, J. A., Ronfeldt, H. M., Calhoun, K. S., \& Arias, I. (1998). Prevalence of traumatic events and peritraumatic predictors of posttraumatic stress symptoms in a nonclinical sample of college students. Journal of Traumatic Stress, 11(4), 645-664.

Breakwell, G., Hammond, S.M., Fife-Schaw, C. \& Smith, J.A. (Eds.). (2006). Research methods in psychology. London: Sage.

Briere, J., \& Zaidi, L. Y. (1989). Sexual abuse histories and sequelae in female psychiatric emergency room patients. The American Journal of Psychiatry, 146(12), 1602-1606.

Brooker, C., Tocque, K., Kennedy, A., \& Brown, M. (2016). The care programme approach, sexual violence and clinical practice in mental health. Journal of Forensic and Legal Medicine, 43, 97-101. doi:10.1016/j.jflm.2016.07.011

Bunting, B.P., Murphy, S.D., O’Neill, S.M. \& Ferry, F.R. (2013). Lifetime prevalence of mental health disorders and delay in treatment following initial onset: Evidence from the Northern Ireland Study of Health and Stress. Psychol Medicine, 42(8), 1727-1739. doi: $10.1017 / \mathrm{S} 0033291711002510$

Cavanagh, M., Read, J., \& New, B. (2004). Sexual abuse inquiry and response: A New Zealand training programme. New Zealand Journal of Psychology, 33(3), 137-144. 
Centre for Reviews and Dissemination (CRD, 2008). CRD's guidance for undertaking reviews in health care. Retrieved from https://www.york.ac.uk/media/crd/Systematic_Reviews.pdf. Cunningham, T., Shannon, C., Crothers, I., Hoy, K., Fitzsimmons, C., McCann, R. \& Mulholland, C. (2016). Enquiring about traumatic experiences in psychosis: A comparison of case notes and self-report questionnaires. Psychosis, 8(4), 301-310. doi:10.1080/17522439.2015.1115542

Currier, G. W., Barthauer, L. M., Begier, E., \& Bruce, M. L. (1996). Training and experience of psychiatric residents in identifying domestic violence. Psychiatric Services, 47(5), 529530.

Currier, G. W., \& Briere, J. (2000). Trauma orientation and detection of violence histories in the psychiatric emergency service. The Journal of Nervous and Mental Disease, 188(9), 622624.

Cusack, K. J., Frueh, B. C., \& Brady, K. T. (2004). Trauma history screening in a community mental health center. Psychiatric Services, 55(2), 157-162. doi: 10.1176/appi.ps.55.2.157

Donohoe, J. (2010). Uncovering sexual abuse: evaluation of the effectiveness of The Victims of Violence and Abuse Prevention Programme. Journal of Psychiatric and Mental Health Nursing, 17(1), 9-18. doi: 10.1111/j.1365-2850.2009.01479.x

Elhai, J.D., Ford, J.D \& Naifeh, J.A. (2010). Assessing trauma exposure and posttraumatic morbidity. In G.M. Rosen and B. Christopher Frueh (Eds). Clinician's guide to Posttraumatic Stress Disorder. (pp 119 -151). New Jersey: John Wiley \& Sons.

Elhai, J. D., Gray, M. J., Kashdan, T. B., \& Franklin, C. L. (2005). Which instruments are most commonly used to assess traumatic event exposure and posttraumatic effects?: A survey of 
traumatic stress professionals. Journal of Traumatic Stress, 18, 541-545. doi: $10.1002 /$ jts. 20062

Frans, Ö., Rimmö, P. A., Åberg, L., \& Fredrikson, M. (2005). Trauma exposure and posttraumatic stress disorder in the general population. Acta Psychiatrica Scandinavica, 111(4), 291-290. doi: 10.1111/j.1600-0447.2004.00463.x

Grey Literature Report. (n.d.). Retrieved December 3d, 2017, from http://www.greylit.org/. Hepworth, I. \& McGowan, L. (2012). Do mental health professionals enquire about childhood sexual abuse during routine mental health assessment in acute mental health settings? A substantive literature review. Journal of Psychiatric and Mental Health Nursing, 20(6), 473-483. doi:10.1111/j.1365-2850.2012.01939.x

Kessler, R. C., Sonnega, A., Bromet, E., Hughes, M., \& Nelson, C. B. (1995). Posttraumatic stress disorder in the National Comorbidity Survey. Archives of General Psychiatry, 52(12), 10481060.

Krinsley, K.E., Gallagher, J.G., Weathers, F.W., Kutter, C.J. \& Kaloupek, D.G. (2003).

Consistency of retrospective reporting about exposure to traumatic events. Journal of Traumatic Stress, 16(4), 399-409.

Liberati, A., Altman, D. G., Tetzlaff, J., Mulrow, C., Gøtzsche, P. C., Ioannidis, J. P., ... \& Moher, D. (2009). The PRISMA statement for reporting systematic reviews and meta-analyses of studies that evaluate health care interventions: explanation and elaboration. PLoS Medicine, 6(7). doi: 10.1371/journal.p,ed.1000100

Lotzin, A., Buth, S., Sehner, S., Hiller, P., Martens, M. S., Pawils, S., ... \& Schäfer, I. (2018). "Learning how to ask": Effectiveness of a training for trauma inquiry and response in 
substance use disorder healthcare professionals. Psychological Trauma: Theory, Research, Practice, and Policy, 10(2), 229. doi: 10.1037/tra0000269.supp

Mansfield, Y., Meehan, T., Forward, R., \& Richardson-Clarke, F. (2017). Asking the Question: Childhood Sexual Abuse in Adults with Mental Illness. Australian Social Work, 70(3), 363-371. doi: 1080/0312407X.2016.1213873

McHugh, M. L. (2012). Interrater reliability: the kappa statistic. Biochemia medica, 22(3), 276282.

Mitchell, B. S. N., \& Grindel, C. G \& Laurenzano, C. (1996). Sexual Abuse Assessment on Admission by Nursing Staff in General Hospital Psychiatric Settings. Psychiatric Services, 47(2), 159 - 165 .

Murray, C. E., Davis, J., Rudolph, L., Graves, K. N., Colbert, R., Fryer, M., ... \& Thigpen, B. (2016). Domestic violence training experiences and needs among mental health professionals: Implications from a statewide survey. Violence and Victims, 31(5), 901-920. doi: 10.1891/0886-6708.VV-D-14-00092

National Heart, Lung \& Blood Institute (NHLBI, 2014a). Quality Assessment Tool for BeforeAfter (Pre-Post) Studies With No Control Group. Retrieved from https://www.nhlbi.nih.gov/health-pro/guidelines/in-develop/cardiovascular-riskreduction/tools/before-after

National Heart, Lung \& Blood Institute (NHLBI, 2014b).Quality Assessment Tool for Observational Cohort and Cross-Sectional Studies. Retrieved from https://www.nhlbi.nih.gov/health-pro/guidelines/in-develop/cardiovascular-riskreduction/tools/cohort 
Read, J., \& Fraser, A. (1998). Abuse histories of psychiatric inpatients: to ask or not to ask?. Psychiatric Services, 49(3), 355-359. doi: 10.1176/ps.49.3.355

Read, J, Hammersley, P. \& Rudegeair, T. (2007). Why, when and how to ask about childhood abuse. Advances in Psychiatric Treatment, 13, 101-110. doi: 10.1192/apt.bp.106.002840

Read, J., Harper, D., Tucker, I. \& Kennedy, A. (2018a). Do adult mental health services identify child abuse and neglect? A systematic review. International Journal of Mental Health Nursing, 27, 7-19. doi: 10.1111/inm.12369

Read, J., Harper, D., Tucker, I. \& Kennedy, A. (2018b). How do mental health services respond when child abuse or neglect become known? A literature review. International Journal of Mental Health Nursing, 27(6), 1606-1617. doi.org/10.1111/inm.12498

Rossiter, A., Byrne, F., Wota, A.P., Nisar, Z., Ofuafor, T., Murray, I., Byrne, C. \& Hallahan, B. (2015). Childhood trauma levels in individuals attending adult mental health services: An evaluation of clinical records and structured measurements of childhood trauma. Child Abuse \& Neglect, 44, 36-45. doi: 10.1016/j.chiabu.2015.01.001

Ruijne, R. E., Howard, L. M., Trevillion, K., Jongejan, F. E., Garofalo, C., Bogaerts, S., ... \& Kamperman, A. M. (2017). Detection of domestic violence by community mental health teams: a multi-center, cluster randomized controlled trial. BMC Psychiatry, 17(1), 288. doi: $10.1186 / \mathrm{s} 12888-017-1399-7$

Sampson, M., \& Read, J. (2017). Are mental health staff getting better at asking about abuse and neglect? International Journal of Mental Health Nursing, 26(1), 95-104. doi: $10.1111 /$ inm. 12237

Scottish Intercollegiate Guidelines Network checklists (SIGN, 2011). Notes on the use of methodology checklist: Randomised Control Trials, Cohort Studies, Systematic Reviews 
and Meta-analyses, Case Control Studies. Retrieved from https://staticcontent.springer.com/esm/art\%3A10.1186\%2F2046-4053-117/MediaObjects/13643_2011_18_MOESM3_ESM.PDF

Shannon, C., Maguire, C., Anderson, J., Meenagh, C. \& Mulholland, C. (2011). Enquiring about traumatic experiences in bipolar disorder: A case note and self-report comparison. Journal of Affective Disorders, 133, 352-355. doi: 10.1016/j.jad.2011.04.022

Toner, J., Daiches, A., \& Larkin, W. (2013). Asking about trauma: the experiences of psychological therapists in early intervention services. Psychosis, 5(2), 175-186. doi:10.1080/17522439.2012.697484

von Elm, E., Altman, D.G., Gøtzsche5,P.C.,Mulrow, C.D., Pocock, S.J.,Poole, C., Schlesselman, J.J., Egger, M. \& Vandenbroucke1, J.P. (2007). Strengthening the Reporting of Observational Studies in Epidemiology (STROBE): Explanation and Elaboration. Retrieved from http://journals.plos.org/plosmedicine/article/file?id $=10.1371 /$ journal.pmed.0040296\&type $=$ printable

Vrana, S., \& Lauterbach, D. (1994). Prevalence of traumatic events and post-traumatic psychological symptoms in a nonclinical sample of college students. Journal of Traumatic Stress, 7(2), 289-302.

Walters, S., Hogg, L., \& Gillmore, C. (2016). Evaluation of a tailored training programme to improve the assessment and treatment of trauma in an Early Intervention in Psychosis (EIP) service. Psychosis, 8(3), 226-237. doi:10.1080/17522439.2015.1131324

Young, M., Read, J., Barker-Collo, S., \& Harrison, R. (2001). Evaluating and overcoming barriers to taking abuse histories. Professional Psychology: Research and Practice, 32(4), 407. doi: 10.1037/0735-7028.32.4.407 


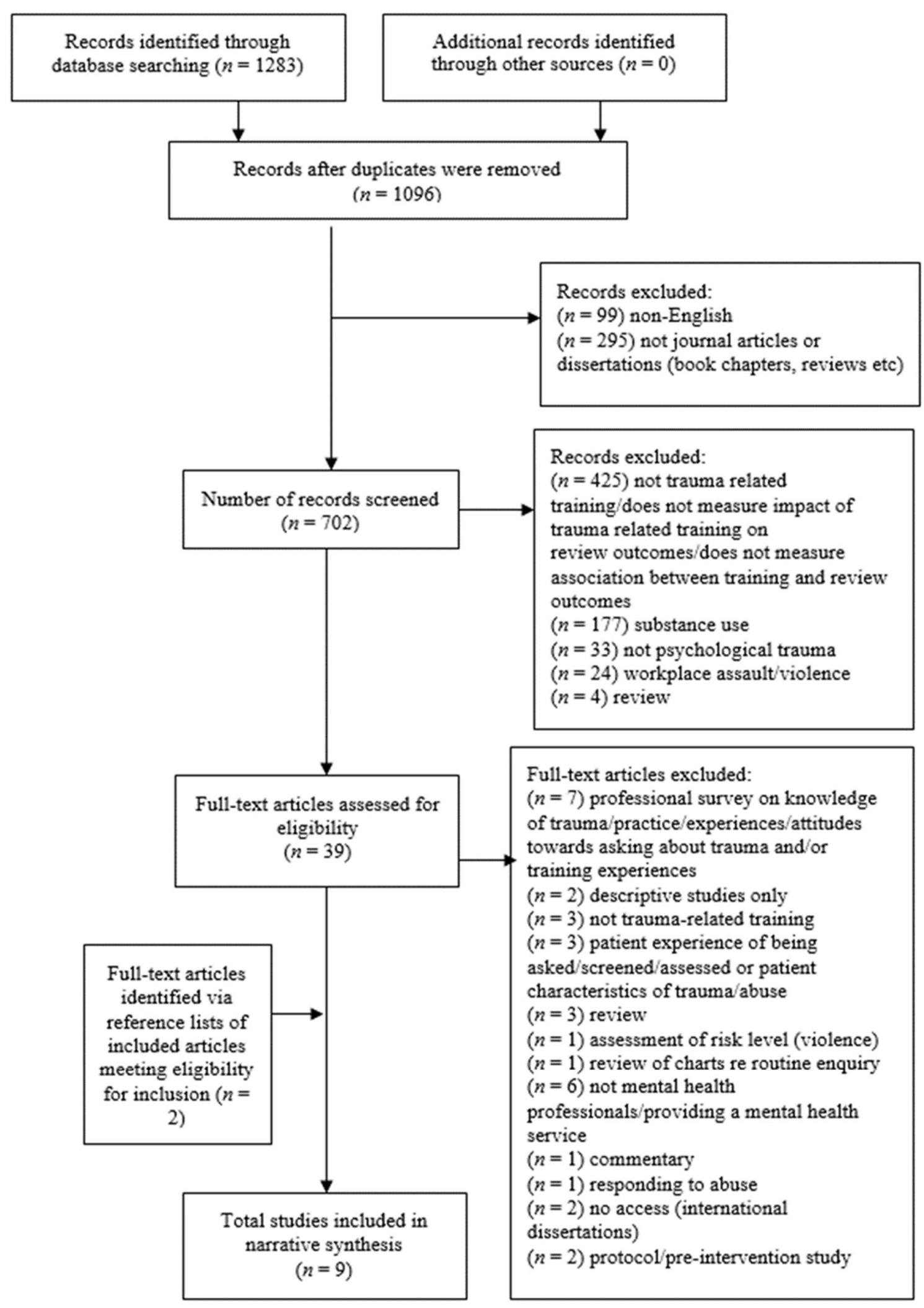

Figure 1. PRISMA flow diagram of search process. Adapted from Liberati et al. (2009) 
Quality Assessment tool

\section{Score Key}

$2=$ Well Addressed

$1=$ Adequately Addressed

$0=$ Poorly addressed, not addressed, not reported, not applicable

\section{Criteria}

Score

1. To what extent was the study question or objectives clearly stated? Did the authors describe their goal in conducting this research? Is it easy to understand what they were looking to find?

2. To what extent was the study population clearly described and defined? Did the authors describe the group of people from which the study participants were selected or recruited, using demographics, location and time period?

3. To what extent were issues relating to sample size addressed? Did the authors present their reasons for selecting or recruiting the number of people included or analyzed? Do they note or discuss the statistical power of the study/conduct sample size calculation?

4. To what extent was the intervention/test/exposure sufficiently defined or described in the study?

Duration/timing of intervention, details as to what the intervention involved

5. To what extent were the tools or methods used to measure the outcomes (dependent variables) clearly defined, prespecified, accurate and reliable-for example, have they been validated or are they objective?

6. To what extent was the timeframe sufficient so that one could reasonably expect to see an association between intervention/exposure (i.e training) and outcome if it existed?

7. To what extent was the following achieved in the discussion section of the study:

Summary of key results with reference to study objectives, discuss limitations of the study, taking into account sources of bias or imprecision, discuss both direction and magnitude of any potential bias, give a cautious overall interpretation of results considering objectives, limitations, multiplicity of analyses, results 
from similar studies, and other relevant evidence, discuss the generalisability (external validity) of the study results

8. To what extent were key potential confounding variables controlled for? Statistical analysis, design, counterbalancing, random sampling/allocation etc

9. To what extent were statistics and statistical tests described, appropriate and reported? e.g. effect sizes, tests named, means and SD's provided etc 
Table 1

Quality appraisal scores and categories

\begin{tabular}{|c|c|c|c|c|c|c|c|c|c|c|}
\hline Study & Aims & Population & $\begin{array}{l}\text { Sample } \\
\text { size }\end{array}$ & $\begin{array}{l}\text { Intervention } \\
\text { exposure }\end{array}$ & Outcomes & $\begin{array}{l}\text { Time } \\
\text { frame }\end{array}$ & Discussion & Confounding & Statistics & $\begin{array}{l}\text { Quality } \\
\text { Category }\end{array}$ \\
\hline $\begin{array}{l}\text { Cavanagh } \\
\text { et al. } \\
(2004)\end{array}$ & 2 & 2 & 1 & 2 & 1 & 0 & 1 & 1 & 1 & Fair \\
\hline $\begin{array}{l}\text { Sampson } \\
\text { \& Read } \\
(2017)\end{array}$ & 2 & 2 & 1 & 1 & 1 & 1 & 2 & 1 & 2 & Fair \\
\hline $\begin{array}{l}\text { Lotzin et } \\
\text { al. (2018) }\end{array}$ & 2 & 2 & 2 & 2 & 1 & 2 & 2 & 2 & 2 & Good \\
\hline $\begin{array}{l}\text { Donohoe } \\
(2010)\end{array}$ & 2 & 0 & 1 & 1 & 0 & 0 & 2 & 0 & 0 & Poor \\
\hline
\end{tabular}




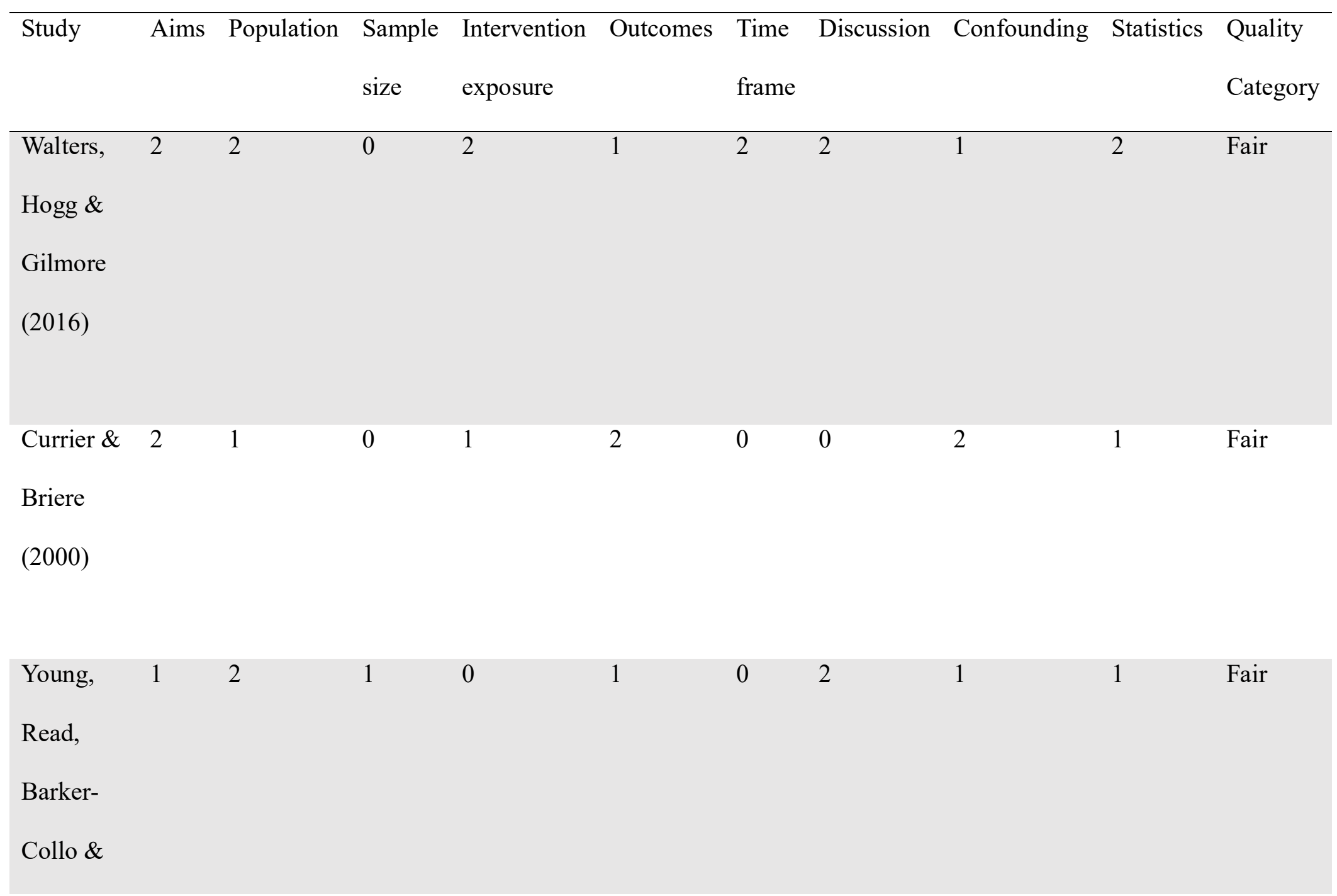




\begin{tabular}{|c|c|c|c|c|c|c|c|c|c|c|}
\hline Study & Aims & Population & $\begin{array}{l}\text { Sample } \\
\text { size }\end{array}$ & $\begin{array}{l}\text { Intervention } \\
\text { exposure }\end{array}$ & Outcomes & $\begin{array}{l}\text { Time } \\
\text { frame }\end{array}$ & Discussion & Confounding & Statistics & $\begin{array}{l}\text { Quality } \\
\text { Category }\end{array}$ \\
\hline $\begin{array}{l}\text { Harrison } \\
(2001)\end{array}$ & & & & & & & & & & \\
\hline $\begin{array}{l}\text { Murray et } \\
\text { al. (2016) }\end{array}$ & 1 & 2 & 1 & 0 & 0 & 0 & 2 & 1 & 2 & Fair \\
\hline Currier, & 2 & 1 & 1 & 0 & 0 & 1 & 1 & 0 & 0 & Poor \\
\hline Barthauer, & & & & & & & & & & \\
\hline Begier \& & & & & & & & & & & \\
\hline $\begin{array}{l}\text { Bruce } \\
\text { (1996) }\end{array}$ & & & & & & & & & & \\
\hline
\end{tabular}


Table 2

Summary of key results

\begin{tabular}{|c|c|c|c|c|}
\hline $\begin{array}{l}\text { Author and } \\
\text { design }\end{array}$ & $\begin{array}{l}\text { Participants (age, gender } \\
\text { and profession) }\end{array}$ & $\begin{array}{l}\text { Training details (duration, } \\
\text { format, type of trauma covered, } \\
\text { content) }\end{array}$ & $\begin{array}{l}\text { Data collection } \\
\text { instruments }\end{array}$ & Key results \\
\hline Cavanagh et al. & $N=85.53 \%$ nurses, & One-day group training & Self-report & No statistically significant \\
\hline$(2004)$ & remainder & programme (New Zealand & questionnaire pre & self-reported change in the \\
\hline Quasi- & therapist/psychotherapists, & training programme), focusing & and six weeks & number of client cases in \\
\hline experimental & psychologists, social & on childhood physical and & post- training $(n=$ & which clinicians believed \\
\hline \multirow[t]{7}{*}{ (pre-post). } & workers, occupational & sexual abuse. Consisted of & $31)$ & that they knew whether or \\
\hline & therapists, psychiatrists & group discussion, educational & & not there was an abuse \\
\hline & and support workers. & components (research & & history (i.e. no statistically \\
\hline & Majority aged 30-39 years. & summary, link between abuse & & significant change in self- \\
\hline & $73 \%$ female; $27 \%$ male. & and mental health, prevalence & & reported detection). \\
\hline & & and effects of abuse) and & & (Unable to detect effect \\
\hline & & practical skills. & & size). \\
\hline
\end{tabular}




\begin{tabular}{|c|c|c|c|c|}
\hline $\begin{array}{l}\text { Author and } \\
\text { design }\end{array}$ & $\begin{array}{l}\text { Participants (age, gender } \\
\text { and profession) }\end{array}$ & $\begin{array}{l}\text { Training details (duration, } \\
\text { format, type of trauma covered, } \\
\text { content) }\end{array}$ & $\begin{array}{l}\text { Data collection } \\
\text { instruments }\end{array}$ & Key results \\
\hline Sampson and & Initial assessments & A one-day group training & File audit of 250 & Statistically significant \\
\hline Read (2017) & conducted by psychiatrists, & programme as described in & randomly selected & increases of recorded child \\
\hline Quasi- & nurses, psychologists and & Cavanagh et al. (2004). This & files. Comparison & sexual abuse $(\Phi=0.13)$ \\
\hline experimental/file & other. $62 \%$ of female; $38 \%$ & training was delivered several & of detection rates & child physical abuse $(\Phi=$ \\
\hline \multirow[t]{3}{*}{ audit analysis } & male. & times annually for approx. nine & to 1997 audit of & 0.22 ), adult sexual abuse \\
\hline & & years. & 200 files (Agar et & $(\Phi=0.12)$ but not adult \\
\hline & & & al., 2002). & physical assault $(\Phi=0.06)$ \\
\hline Lotzin et al. 2018 & $N=148.70 .2 \%$ of sample & One-day group programme & Self-report & Self-report change of \\
\hline Cluster & were social pedagogues, & with refresher session three & questionnaire at & frequency of inquiry about \\
\hline randomised & remainder were & months post training in relation & baseline $(n=$ & trauma was significantly \\
\hline control design. & pedagogues, psychologist, & to neglect, physical sexual and & 132), 3-month (n & greater (from baseline) at \\
\hline
\end{tabular}




\begin{tabular}{|c|c|c|c|c|}
\hline $\begin{array}{l}\text { Author and } \\
\text { design }\end{array}$ & $\begin{array}{l}\text { Participants (age, gender } \\
\text { and profession) }\end{array}$ & $\begin{array}{l}\text { Training details (duration, } \\
\text { format, type of trauma covered, } \\
\text { content) }\end{array}$ & $\begin{array}{l}\text { Data collection } \\
\text { instruments }\end{array}$ & Key results \\
\hline & trainees and "other" & emotional abuse (i.e. "Learning & $=104)$ and 6- & three month and six month \\
\hline & recruited from substance & how to ask" training (Read et & month follow-up & follow-up in the \\
\hline & use service. Age of & al., 2007) based on the New & $(n=74)$ & intervention group than \\
\hline & intervention group in years & Zealand training programme, & & controls $(B=0.43)$. No \\
\hline & $(M=42.9) ;$ age of control & Service providers randomly & & significant change from \\
\hline & group in years $(M=42.0)$. & allocated to intervention $(n=$ & & three month to six month \\
\hline & Female $(n=79)$; male $(n=$ & $72)$ or control $(n=76)$. & & follow- up $(B=0.02)$ \\
\hline & $52)$. & & & \\
\hline Donohoe (2010) & $N=53$. Staff nurses, ward & The Victims of Violence and & Self-report & $77 \%$ of professionals felt \\
\hline \multirow[t]{4}{*}{ Survey evaluation } & management, social & Abuse Prevention Programme & questionnaires ( $n$ & that the training had \\
\hline & workers, community & consisted of a one-day group & $=30)$. & changed their practice with \\
\hline & psychiatric nurses, & training and education & & over $35 \%$ of these \\
\hline & occupational therapists, & programme to improve inquiry & & professionals reporting \\
\hline
\end{tabular}




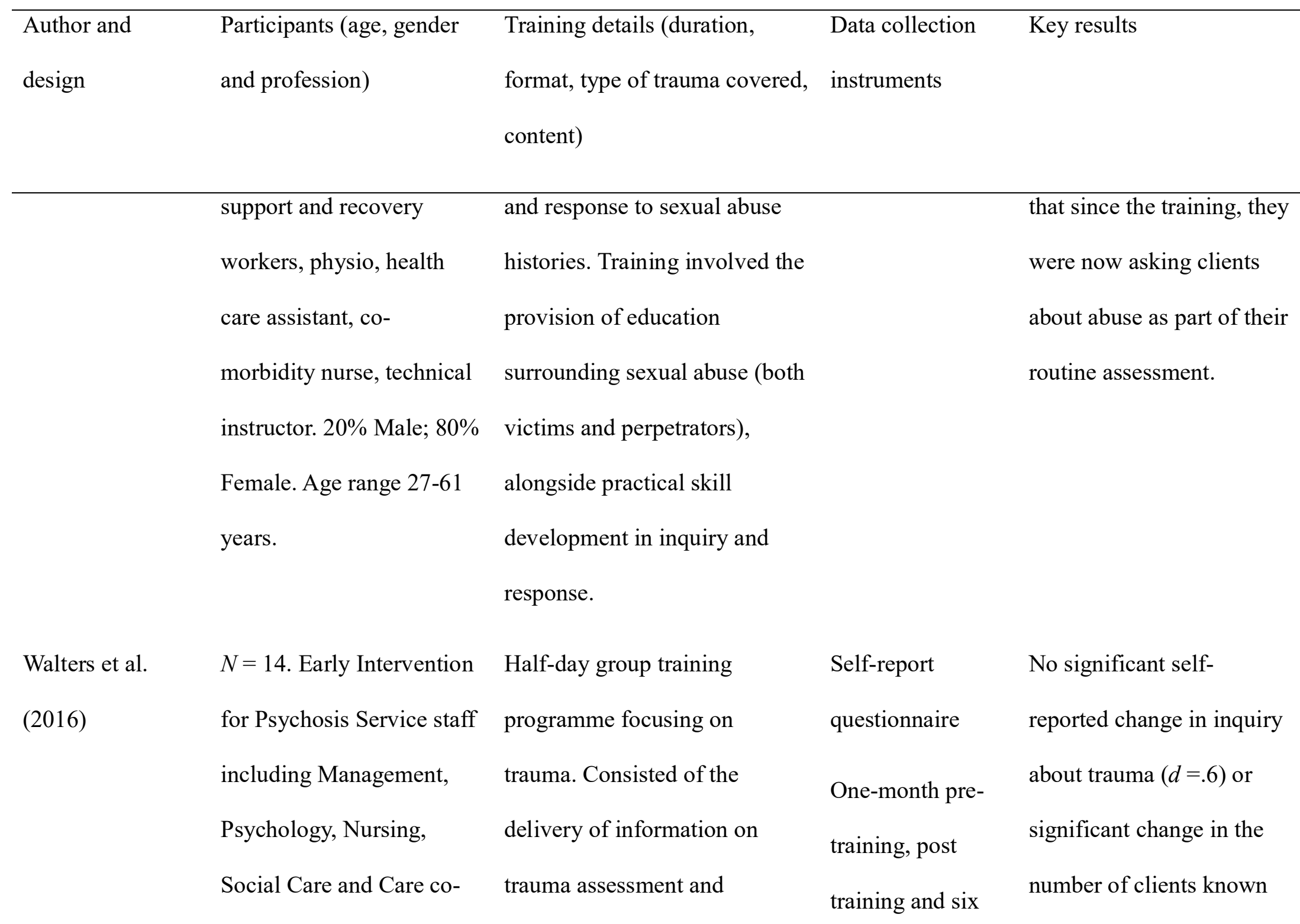




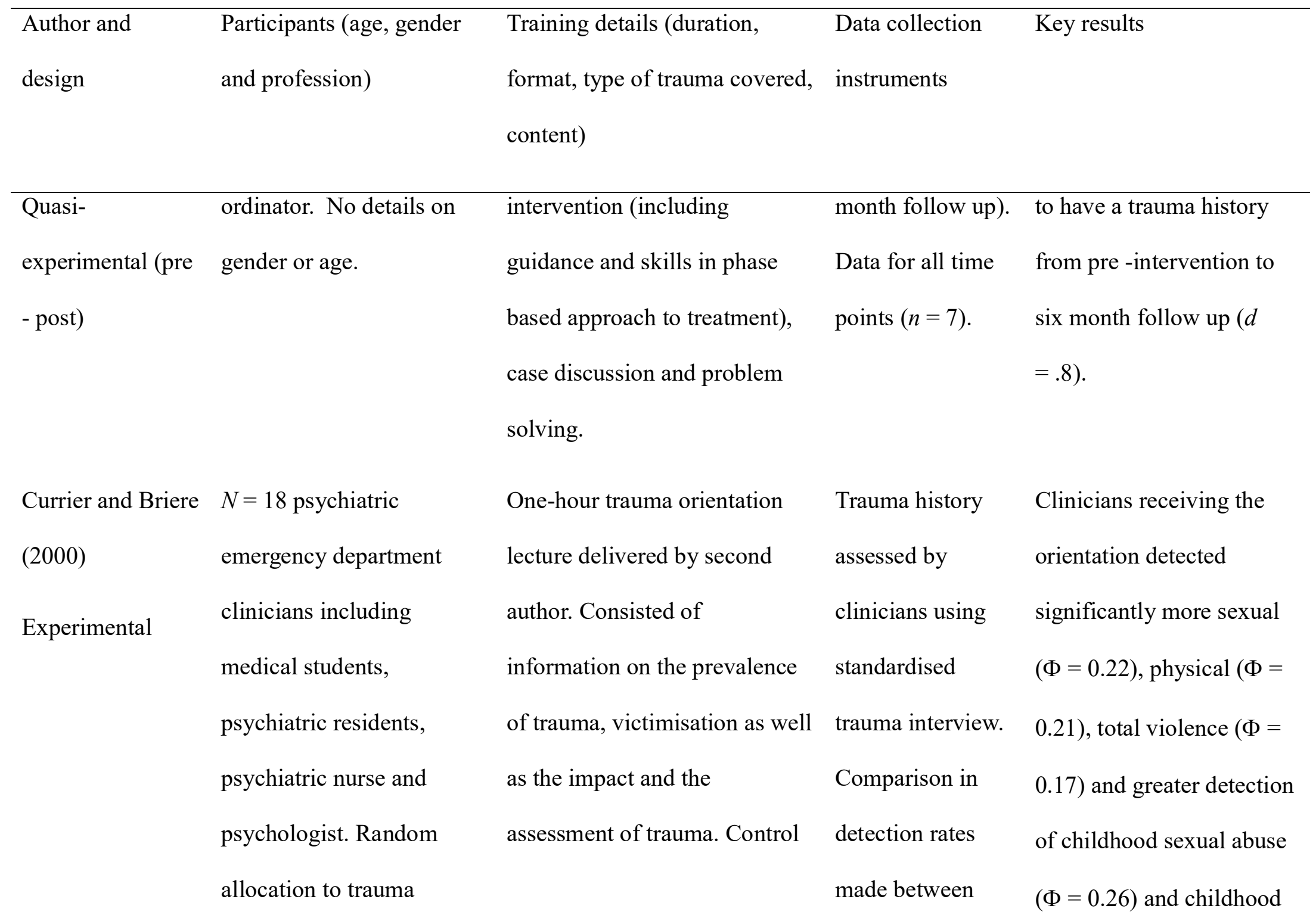




\begin{tabular}{|c|c|c|c|c|}
\hline $\begin{array}{l}\text { Author and } \\
\text { design }\end{array}$ & $\begin{array}{l}\text { Participants (age, gender } \\
\text { and profession) }\end{array}$ & $\begin{array}{l}\text { Training details (duration, } \\
\text { format, type of trauma covered, } \\
\text { content) }\end{array}$ & $\begin{array}{l}\text { Data collection } \\
\text { instruments }\end{array}$ & Key results \\
\hline & $\begin{array}{l}\text { orientation }(n=10) \text { or } \\
\text { control }(n=8) \text {. No details } \\
\text { on gender or age. }\end{array}$ & $\begin{array}{l}\text { group received no information } \\
\text { on trauma. }\end{array}$ & $\begin{array}{l}\text { those who } \\
\text { received training } \\
\text { and control. }\end{array}$ & $\begin{array}{l}\text { physical abuse }(\Phi=0.16) \text {. } \\
\text { No significant difference } \\
\text { between groups in } \\
\text { detection of } \\
\text { childhood/adolescent peer } \\
\text { sexual assault }(\Phi=0.06) \text {, } \\
\text { adult spouse abuse }(\Phi= \\
0.08) \text {, adult non-intimate } \\
\text { physical assault ( } \Phi=0.11) \\
\text { or adult sexual assault }(\Phi \\
=0.14) \text {. }\end{array}$ \\
\hline
\end{tabular}




\begin{tabular}{|c|c|c|c|c|}
\hline $\begin{array}{l}\text { Author and } \\
\text { design }\end{array}$ & $\begin{array}{l}\text { Participants (age, gender } \\
\text { and profession) }\end{array}$ & $\begin{array}{l}\text { Training details (duration, } \\
\text { format, type of trauma covered, } \\
\text { content) }\end{array}$ & $\begin{array}{l}\text { Data collection } \\
\text { instruments }\end{array}$ & Key results \\
\hline Young et al. & $N=144$. Psychologists & Participants were asked via & Self-report & Participants with training \\
\hline$(2001)$ & and Psychiatrists & survey item "Have you ever & questionnaire and & (76\%) were significantly \\
\hline \multirow[t]{4}{*}{ Cross-sectional } & $55 \%$ female; $45 \%$ male & received any training regarding & case vignette. & more likely to ask about \\
\hline & & how to enquire about abuse?" & & abuse in a case vignette ( $g$ \\
\hline & Age $(M=43 ; S D=10.3)$ & No details on content of & & $=0.75)$ \\
\hline & & training available. & & \\
\hline Murray et al. & $N=173$. Marriage and & Over seventy-eight percent of & Self-report & Professionals with training \\
\hline (2016) & Family Therapists & participants had domestic & survey. & were statistically more \\
\hline \multirow{5}{*}{ Cross-sectional } & (41.6\%). Remaining & violence training. For the & & likely to screen all clients \\
\hline & sample included Clinical & majority of these professionals, & & for domestic violence $(\Phi=$ \\
\hline & Social Workers, & this training was eight hours or & & $0.22)$, screen for partner \\
\hline & Psychologists, & less, $\leq 2$ years ago. Over & & sexual abuse $(\Phi=0.19)$ \\
\hline & Counsellors, Substance & ninety-four percent of & & screen for trauma \\
\hline
\end{tabular}




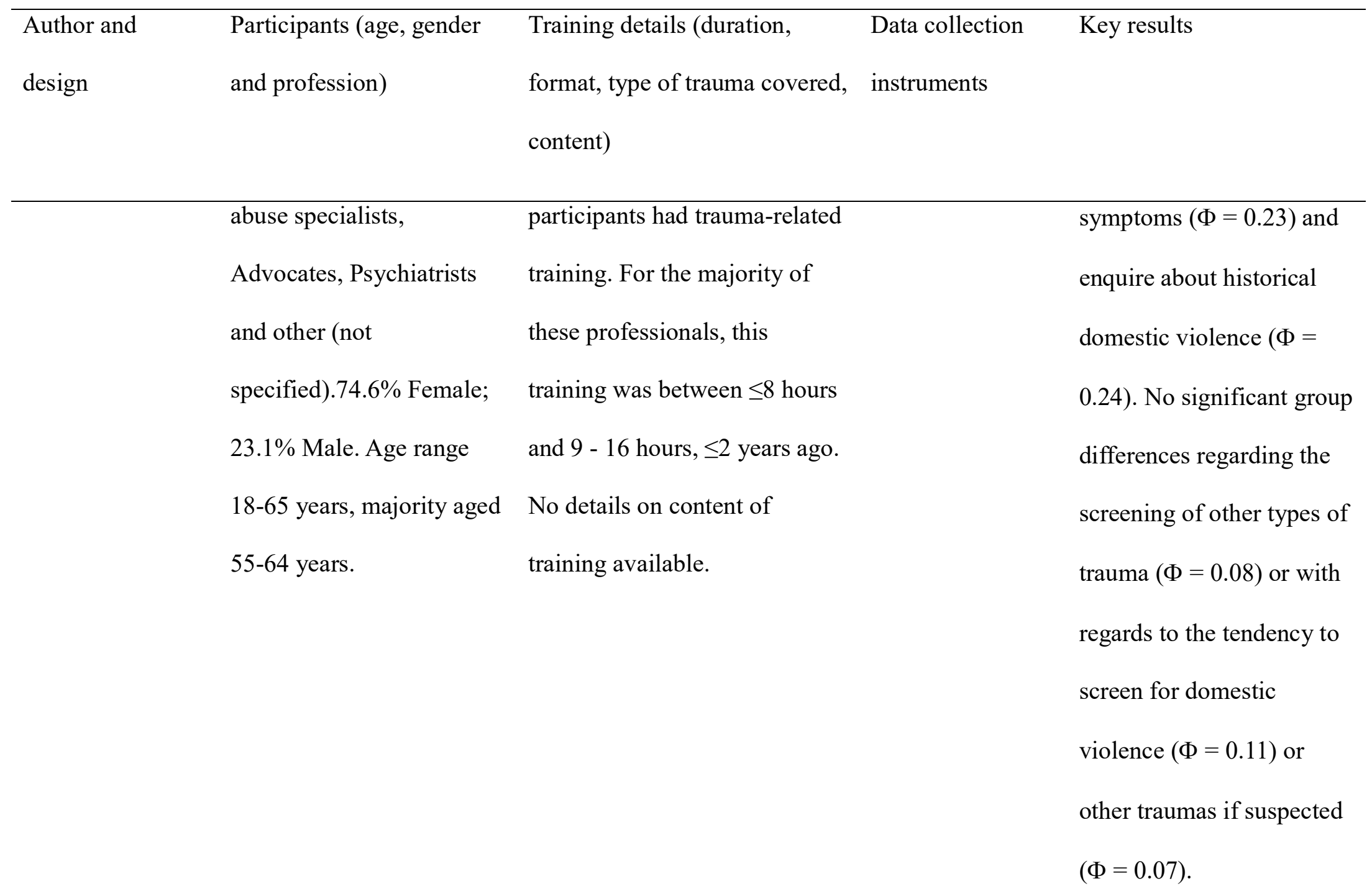




\begin{tabular}{|c|c|c|c|c|}
\hline $\begin{array}{l}\text { Author and } \\
\text { design }\end{array}$ & $\begin{array}{l}\text { Participants (age, gender } \\
\text { and profession) }\end{array}$ & $\begin{array}{l}\text { Training details (duration, } \\
\text { format, type of trauma covered, } \\
\text { content) }\end{array}$ & $\begin{array}{l}\text { Data collection } \\
\text { instruments }\end{array}$ & Key results \\
\hline Currier et al. & $N=145$ psychiatric & Twenty-eight percent had & Survey & Case detection was \\
\hline (1996) & residents at 4 medical & received training on domestic & & significantly correlated \\
\hline \multirow{8}{*}{ Cross-sectional } & schools. No details on & violence, either in medical & & with training in domestic \\
\hline & gender or age- & school (13\%), during residency & & violence $(r=.30) .88 \%$ of \\
\hline & & $(19 \%)$ and from other sources & & those with training \\
\hline & & $(8 \%)$. Between 9.9 and 11.3 & & detected six or more cases \\
\hline & & hours of training on domestic & & of domestic violence in the \\
\hline & & violence received. No details & & past year in comparison to \\
\hline & & on content of training & & $48 \%$ of those who had not \\
\hline & & available. & & $(\Phi=0.35)$ \\
\hline
\end{tabular}


